Rev. Biol. Trop., 47(3): 493-502, 1999

www.ucr.ac.cr. www.ots.ac.cr www.ots.duke.edu

\title{
Zoogeografía de los gastrópodos terrestres de la región oriental de San Luis Potosí, México
}

\author{
Alfonso Correa Sandoval \\ Instituto Tecnológico de Ciudad Victoria, Departamento de Biología Apdo. Postal 175, C. P. 87010, Ciudad Victoria, \\ Tamaulipas, México. Fax: (131) 3-06-63 \\ Correo eléctronico : agutierr@rectoria.uat.mx \\ snailacs@tamps1.telmex.net.mx
}

Recibido 7-IX-1998. Corregido 11-I-1999. Aceptado 2-II-1999.

\begin{abstract}
Abrstract: The land snails of the eastern region of San Luis Potosí, México were surveyed (June, 1995 August, 1996). This region has much topographic and climatic variation. Samples (161) were obtained in 48 localities. The main zoogeographic groups of were the endemic (32.18\%: 28 species and three subspecies) and the neotropical (27.59\%: 24 species and five subspecies) gastropods. The family Spiraxidae has the largest number of endemic species (16). The Eastern Sierra Madre has the largest number of species in the region.
\end{abstract}

Key words: Land snails, survey, biogeography, Mexico.

La Huasteca Potosina, región oriental del Estado de San Luis Potosí, es una de las que posee más variantes topográficas y climáticas en el noreste de México, (Rzedowski 1978, Anónimo 1980, 1981a, 1981b), las cuales pueden influir sobre la distribución y diversidad de los gastrópodos terrestres.

Sin embargo, los estudios zoogeográficos sobre los moluscos terrestres de esta zona, y en general de México, son muy escasos.

Los trabajos de Fischer y Crosse (18701902), Martens (1890) y Pilsbry (1948) establecen que ciertas regiones del país poseen géneros y especies representativos.

En otro estudio, Pilsbry (1903) señaló que la fauna de las localidades de Monterrey y El Diente en Nuevo León, y Ciudad Victoria, en Tamaulipas, confirma la extensión norteña de la región neotropical en el este de México. Más recientemente en el trabajo realizado por Correa (1993) en el municipio de Santiago, Nue- vo León, se concluye que el endemismo es el principal componente zoogeográfico. En Iturbide, Nuevo León, lo es la distribución panamericana (Correa 1996-1997).

Se presentan en esta investigación resultados sobre los ámbitos de distribución geográfica en el Continente Americano de los gastrópodos terrestres de la región oriental de San Luis Potosí, México.

\section{MATERIALES Y MÉTODOS}

Las especies consideradas en este trabajo son las indicadas para el área de estudio $\left(21^{\circ}\right.$ $11^{\prime} 55^{\prime \prime}$ y $23^{\circ} 14^{\prime} 03 \mathrm{~N}$; $98^{\circ} 17^{\prime} 53^{\prime \prime}$ y $100^{\circ} 23^{\prime}$ 39 W, Fig. 1) por Correa et al. (1998). De acuerdo al mismo autor se visitaron 48 localidades para realizar los muestreos, los cuales se hicieron en cuadrantes o parcelas de $1 \mathrm{~m}^{2}$. Se realizaron 161 muestreos en total siguiendo las 


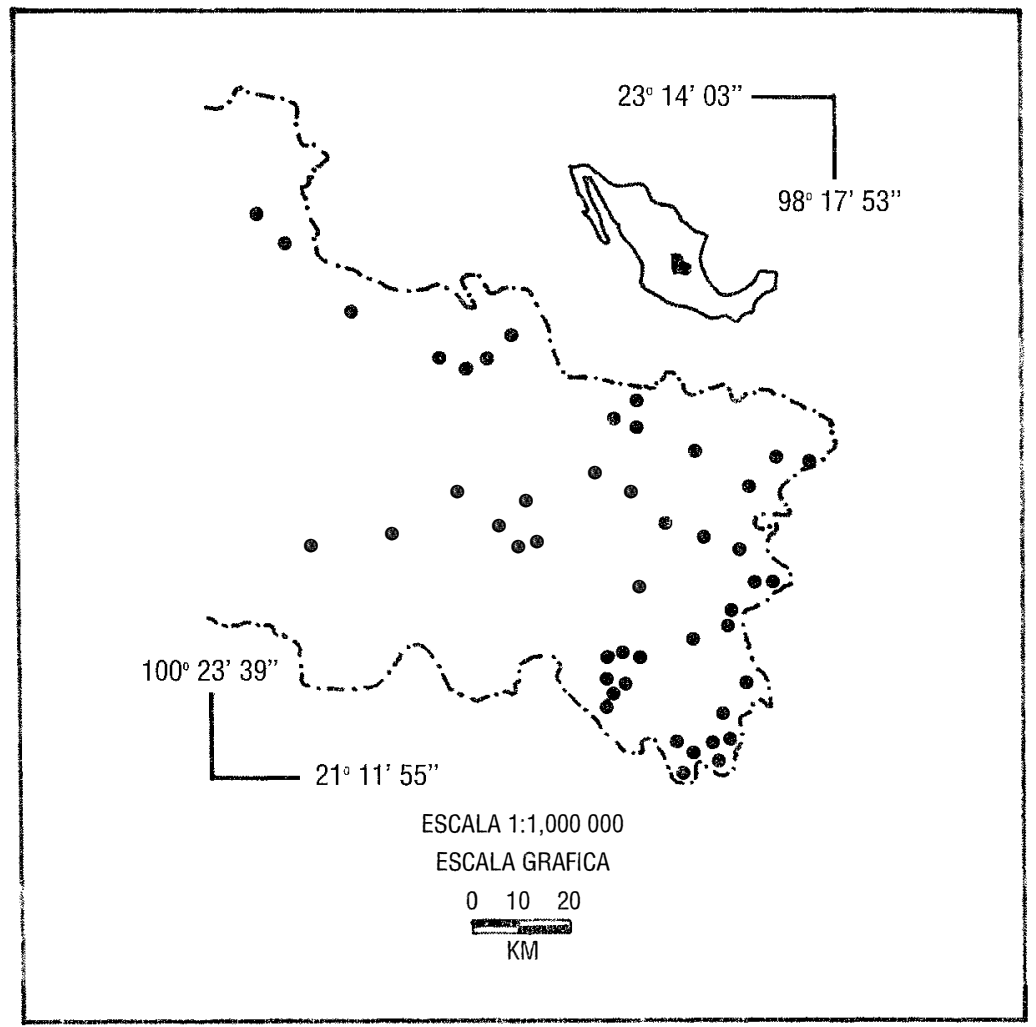

Fig. 1. Localidades de muestreo en la Huasteca Potosina, México.

recomendaciones de Coney et al. (1981), Lavelle et al. (1981) y Pérez et al. (1996), durante los meses de junio de 1995 a agosto de 1996. En cada sitio de muestreo se recogieron en forma directa ejemplares macroscópicos y se obtuvieron muestras de suelo para tamizar y revisar en el laboratorio, de acuerdo a Cameron y Redfern (1976). El tiempo aproximado de muestreo en cada cuadrante fue de una hora. En cada localidad, además de los muestreos, se realizaron recolectas directas adicionales de ejemplares con el fin de complementar la lista de especies. También se revisó la Colección de Moluscos Terrestres (zona San Luis Potosí) del Instituto Tecnológico de Cd. Victoria, Tams. (ITCVZ). De los muestreos se revisaron un total de 1016 lotes (8331 ejemplares) y de la colección ITCVZ 643 lotes (3215 ejemplares). Se revisaron en total 1659 lotes (11,546 ejemplares).
Los ejemplares de todas las especies encontradas en el área de estudio pertenecen a las colecciones malacológicas del Instituto Tecnológico de Cd. Victoria, Tamps. y Florida Museum of Natural History, University of Florida. Duplicados de varias especies se encuentran en el Instituto de Ciencias del Mar y Limnología e Instituto de Biología de la Úniversidad Nacional Autónoma de México.

En resultados las familias siguen principalmente el ordenamiento de Hubricht (1985) el cual se basa en el Handbuch der Palaeozoologie (Sección Euthyneura) de Zilch (1959-1960), de Taylor y Sohl (1962) en parte y el del Florida Museum of Natural History. Los géneros y especies se ordenaron alfabéticamente. La caracterización zoogeográfica en el Continente Americano es a nivel específico o subespecífico según el caso. El símbolo "NT" índica distribución neotropical y "NA" es distribución 
neártica. La distribución panamericana "PA" es neártica y neotropical y se presenta al norte y sur del Trópico de Cáncer. La predominancia distribucional en alguna de las regiones se señala con " $p$ " (principalmente) enseguida de "NT" o "NA". La distribución especial para San Luis Potosí y/o Tamaulipas se índica "EN". Esta caracterización, así como la distribución específica en México y la presencia en otras regiones del mundo que se indica en la parte de "Distribución geográfica" son de acuerdo a los trabajos de Martens (1890), Pilsbry (1919, 1939, 1940, 1946, 1948, 1953, 1956), Baker (1922, 1923, 1928, 1930), Solem (1956, 1957), Thompson $(1968,1980)$, Cheatum y Fullington (1971, 1973), Bequaert y Miller (1973), Peake (1978), Hubricht (1985), Pérez y López (1993) y Correa (1996-1997).

\section{RESULTADOS}

\section{Helicinidae}

Helicina chrysocheila Binney 1851

Distribución geográfica: NTp y NA. Sureste de Texas, E.U.A.: México: Nuevo León (Santiago), Tamaulipas (González y Tampico), San Luis Potosí (El Abra y al sur de Cd. Valies).

\section{Helicina flavida Menke 1828}

Distribución geográfica: NT. México: Veracruz (Córdova, Atoyac, Jica tepec, Mirador, Misantia, Papantia, San Rafael y Veracruz), Morelos (Cuernavaca), Tabasco (San Juan Bautista, Teapa), Campeche (Ercárcega), Chiapas (Palenque), Quintana Roo (Xpujil): Guatemala.

Helicina orbiculata tropica (Pfeiffer 1852)

Distribición geográfica: NAp y NT. Sur y sureste de E.U.A.; México: Coahuila, Nuevo León (Santiago), Tamitulipas (Tampico), San Luis Potosí (sur de Cd. Valles) Helicina sowerbyana Pfeiffer 1848

Distribución geográfica: NTp y NA. México: Nuevo

León (Santiago), Tamaulipas (Cd. Victoria); Guatemala. Felicina vanatae Pilsbry 1909

Distribución geográfica: EN México: San Luis Potosí (Canoas, Tamasope y sur de Micos)

Helicina zephyrina Ducios 1833

Distribución geográfica: NT. México: Tamaulipas (Tampico). San Luis Potosí (Montañas Alvarez y San Dieguito), Veracruz (Alvarado, Casamaloapan, Huatusco, Jalapa, Miraior, Tejeria, Texolo y Veracnz), Oaxaca (Tuxtepec).

Heicina zephyrina zephyrina Duclos 1833

Distribución geográfica: NT. México: Tanaulipas (Tampico), Sar Luis Potosí (Montañas Alvarez y San
Dieguito), Veracruz (Papantla, Misantla, Mirador y Córdova); Nicaragua.

Schasicheila hidalgoana Dall 1897

Distribución geográfica: NT. México: Tamaulipas (Cd. Victoria), San Luis Potosí (El Abra Montañas Alvarez, San Dieguito, Tamasopo, Cd. Vailes), Veracruz (Jalapa y Misantla), Aguascalientes, Hidalgo (Encarnación); Guatemala.

Schasicheila minuscula (Pfeiffer 1859)

Distribución geográfica: NT. México: Veracruz (Misantla), Puebla (Necaxa); Guatemala.

Schasicheila xanthia Pilsbry 1909

Distribución geográfica: EN. México: San Luis Potosí (Canoas).

\section{Ceresidae}

\section{Ceres nelsoni Dall 1898}

Distribución geográfica: NT. México: Tamaulipas (Gómez Farías), San Luis Potosí (Ahuacatlán, Aquuismón, Tamazunchale y Xilitla), Veracruz.

\section{Cyclophoridae}

Aperostoma mexicanum palmeri (Bartsch y Morrison 1942)

Distribución geográfica: NT. México: Tamaulipas (Chamal, El Cielo, Gómez Farías).

\section{Diplommatinidae}

Adelopoma stolli Martens 1890

Distribución geográfica: NT. México: Tamaulipas (Tampico); Guatemala.

\section{Veronicellidae}

Veronicella moreleti (Crosse y Fischer 1872)

Distribución geográfica: NT. México: San Luis Potosí (Cd. Valles, Micos), Veracruz, Oaxaca (Tehuantepec), Campeche (is. del Carmen), Chiapas (Palenque), Yucatán (Aguada, Mérida); Guatemala; Nicaragua.

\section{Carychinioe}

Carychium mexicanum Pilsbry 1891

Distribución geográfica: PA. Sureste de E. U. A.; México: Nuevo León (El Diente y Santiago;, Tamaulipas (Tampico), Veracruz (Crizaba y Texolo), Puebla; Guatemala.

\section{Pupillidae}

Gastrocoptci contracta (Say 1822)

Distribución gecgráfica: PA. Sureste de Cenadá: sureste, noreste y este de E. U. A.; México: Sonore, Nuevo León (Río Manricio), Tamadipas (Tarupico), Smaba, 
Veracruz (Orizaba), Morelos (Yautepec), Puebla (Necaxa); Cuba y Jamaica.

Gastrocopta corticaria (Say 1816)

Distribución geográfica: NAp y NT. Sureste de Canadá; norte, noreste y sureste de E. U. A.; México: Nuevo León (Río Mauricio); Cuba.

Gastrocopta pellucida (Pfeiffer 1841)

Distribución geográfica: PA. Este, sureste, suroeste, sur y centro de E. U. A.; México: Baja California; Sonora (Arroyo San Rafael, Cerro de Oro, San Bernardo, Sierra Los Embudos, Sierra San Ignacio, Río Mayo, Navajo), Nuevo León (Iturbide y Santiago), Tamaulipas (Cd. Victoria y Tampico), Sinaloa (RíoFuentes y San Blas), San Luis Potosí, Veracruz (Antigua), Hidalgo, Tabasco, Campeche (Champotón y Campeche), Yucatán (Puerto Telchac y Becanchen); Guatemala; Nicaragua; Panamá; Ecuador.

Gastrocopta pentodon (Say 1821)

Distribución geográfica: PA. Canadá; sur, sureste, noreste y este de E. U. A.; México: Chihuahua (Río Piedras Verdes), Nuevo León (Pablillo, Río Mauricio), Tamaulipas (Cd. Victoria), San Luis Potosí (San Dieguito), Puebla; Guatemala.

Pupisoma dioscoricola insigne Pilsbry 1920

Distribución geográfica: NTp y NA. Sureste de Texas, Florida (E. U. A.); México: Nuevo León (Iturbide), San Luis Potosí (Cascadas de Valles, Cueva Choy, Río Gallinas), Veracruz, Yucatán (Izmal, Cenote Santa Ana Valladolid); Centro y Sudamérica; Jamaica, Trinidad, oeste del Caribe.

Pupisoma minus Pilsbry 1920

Distribución geográfica: NTp y NA. Florida (E. U. A.); México: Sonora (Cerro de Oro, Río Mayo, Navajo), Nuevo León (Pablillo), San Luis Potosí (Canoas); Guatemala; Jamaica.

Vertigo ovata Say 1822

Distribución geográfica: NA. Is. Kadiak y Tigalda (Alaska); Ontario, Quebec, Isla Principe Eduardo (Canadá); México: Sonora (Río Sonoyta en Sonoyta, a 5 millas al sur de la frontera mexicana), San Luis Potosí (San Vicente).

\section{Strobilopsidae}

Strobilops aenea mexicana Pilsbry 1903

Distribución geográfica: NTp y NA. México: Nuevo León (El Diente, Monterrey, Río Mauricio), Puebla, Veracruz.

Strobilops hubbardi Brown 1861

Distribución geográfica: PA. Sur y sureste de E. U. A.; México: Tamaulipas (Tampico), Planice costera del noreste; Caribe.

\section{Succineidae}

Succinea concordialis Gould 1848

Distribución geográfica: NAp y NT. Sur y sureste de E: U. A.; México: Veracruz (Tuxpan), San Luis Potosí (Huichihuayán).
Succinea luteola Gould 1848

Distribución geográfica: PA. Suroeste, sur y sureste de E. U. A.; México: Tamaulipas (Tampico), San Luis Potosí (Valles), Veracruz, Puebla (Tehuacán), Guerrero (Venta de Zopilote), Yucatán (Isla Cerro, Ciénega, Progreso); Guatemala.

Succinea panucoensis Pilsbry 1909

Distribución geográfica: EN. México: Tamaulipas (Tampico); San Luis Potosí (Lagunas Catamas, El Lagarto y Pujal).

\section{Discidae}

Gonyodiscus victorianus (Pilsbry 1903)

Distribución geográfica: NT. México: Tamaulipas (Cd. Victoria), Puebla (Necaxa).

\section{Punctidae}

Punctum minutissimum (Lea 1841)

Distribución geográfica: NAp y NT. Noreste y este de E. U. A.; México: Puebla (Necaxa).

\section{Charopidae}

\section{Chanomphalus pilsbryi (Baker 1927)}

Distribución geográfica: NT. México: Tamaulipas (Tampico) Veracruz (Cuatotolapam, Sumidero y Veracruz), Islas Marías, Puebla (Necaxa); Guatemala.

\section{Zonitidae}

Glyphyalinia indentata (Say 1823)

Distribución geográfica: PA. Este, suroeste y sui de E. U. A.; México: Baja California, Sonora, Chihuahua, Nuevo León (Iturbide y Santiago), Durango, Jalisco, Michoacán, Morelos, Puebla; Guatemala; Nicaragua.

Glyphyalina sp. nov.

Distribución geográfica: EN. México: San Luis Potosí (Región Oriental).

Hawaiia minuscula (Binney 1840)

Distribución geográfica: PA. Desde Alaska; Canadá, Maine y Bermudas a Florida, Costa Rica y el Caribe; México: Baja California, Sonora (Cerro de Oro), Tamaulipas (Tampico), San Luis Potosí, Veracruz, Nayarit, Puebla, Campeche (Champotón y Pixtún), Yucatán; Irlanda e Inglaterra; Japón.

Mesomphix montereyensis victoriana (Pilsbry 1903)

Distribución geográfica: EN. México: Tamaulipas (Cd. Victoria y El Cielo).

Zonitoides arboreus (Say 1816)

Distribución geográfica: PA. Estados Unidos de América; México: Chihuahua, Nuevo León (El Diente), Veracruz (Jalapa, Las Vigas, Mirador), Puebla, Morelos; Centroamérica; Caribe; introducido en Japón; Sudafrica; Australia y Hawaii. 


\section{Systrophiidae}

Miradiscops opal (Pilsbry 1919)

Distribución geográfica: NT. México: Veracruz (Córdova), Puebla (Necaxa); Nicaragua; Venezuela.

Miradiscops puncticipitis (Pilsbry 1926)

Distribución geográfica: NT. México: Veracruz (Atoyac y Sumidero), Puebla (Necaxa); Guatemala.

\section{Helicarionidae}

\section{Guppya gundlachi (Pfeiffer 1839)}

Distribución geográfica: NTp y NA. Florida, desde Texas (E. U. A.) a Venezuela; México: Tamaulipas (Cd. Victoria, Tampico), San Luis Potosí (San Dieguito), Campeche (Is. Carmen y Champotón), Quintana Roo (Xpujil, Campeche, Cozumel, San Miguel); Centroamérica; Caribe; Venezuela; Trinidad; Brasil.

Guppya micra Pilsbry 1903

Distribución geográfica: NT. México: Tamaulipas

(Cd. Victoria), Michoacán (Pátzcuaro).

Guppya sterkiia punctum Baker 1930

Distribución geográfica: NT. México: Veracruz, Puebla (Necaxa).

Habroconus elegantula (Pilsbry 1919)

Distribución geográfica: NTp y NA. México: Nuevo León (El Diente e Iturbide), Tamaulipas (Cd. Victoria y Tampico), San Luis Potosí (Cd. Valles), Veracruz (Atoyac, Jalapa, Orizaba, Sumidero), Jalisco (Guadalajara), Michoacán (Uruapan), Puebla (Necaxa), Morelos (Yautepec); Guatemala.

\section{Ferussaciidae}

Cecilioides consobrina veracruzensis (Crosse y Fischer 1877)

Distribución geográfica: NT. México: Tamaulipas (Tampico), Veracruz (Antigua, Veracruz), Yucatán (Chichen-Itza), trópicos de México; Centro América.

Subulinidae

\section{Beckianum beckianum (Pfeiffer 1846)}

Distribución geográfica: NT. México: Tamaulipas (Tampico), San Luis Potosí (Huichihuayán), Veracruz (Lago Catemaco), Tabasco; Guatemala; Nicaragua; Panamá; Caribe; hasta Brasil y Perú.

Lamellaxis gracilis (Hutton1834)

Distribución geográfica: NTp y NA. Suroeste y sureste de E. U. A.; trópicos de ambos hemisferios; México: Baja California, Sonora (Sierra Alamos), San Luis Potosí (El Abra), Campeche (Champotón, Seybaplaya, Campeche, Hopelchén, Dzibalchén), Yucatán (Dzitas, Mérida, Libre Unión), Quintana Roo (Is. Cozumel, San Miguel); Guatemala; Panamá; Cuba, Oeste del Caribe; India.

Lamellaxis micra (Orbigny 1835)

Distribución geográfica: NTp y NA. Florida a Louisiana (E. U. A.); trópicos de América; México: Tamaulipas
(Tampico), Veracruz (Antigua, Misantla), Chiapas, Tabasco (Juan Bautista), Yucatán (Chichen-Itza; Izamal, Progreso, Tekanto, Ticul, Tunkas; Centroamérica; Caribe; Sudamérica.

Leptinaria mexicana (Pfeiffer 1866)

Distribución geográfica: NT. México: Tamaulipas (Tampico), Veracruz (Agua Caliente, Camino del Obispo, Camino de Arroyo Hondo, Coatepec, Jalapa, Misantla, San Eligio y Texolo); Honduras.

Leptinaria tamaulipensis Pilsbry, 1903

Distribución geográfica: NT. México: Tamaulipas (Cd. Victoria, Tampico), San Luis Potosí (Huichihuayán).

Subulina octona (Bruguiere 1792)

Distribución geográfica: NT. Florida (E. U. A.); trópicos de América; México: Veracruz, Tabasco (Teapa), Campeche, (Cd. del Carmen y Campeche), Yucatán (Izamal, Mérida); Centroamérica; Caribe; Brasil; Ecuador; Africa.

Achatinidae

Rumina decollata (Linnaeus 1758)

Distribución geográfica: NAp y NT. Este, sureste y suroeste de E. U. A.; México: Nuevo León (Iturbide), Tamaulipas, San Luis Potosí; Is. Bermuda; Cuba; nativo de la región Mediterránea; Europa; Asia y Africa.

Spiraxidae

Coelostele tampicoensis Pilsbry 1906

Distribución geográfica: EN. México: Tamaulipas (Tampico)

Euglandina corneola (Binney 1857)

Distribución geográfica: EN. México: Tamaulipas (Tampico), San Luis Potosí (Cd. Valles).

Euglandina cymatophora Pilsbry 1909

Distribución geográfica: EN. México: San Luis Potosí (Canoas).

Euglandina lamyi (Fischer y Chatelet 1903)

Distribución geográfica: EN. México: San Luis Potosí (Cárdenas).

Euglandina oblonga potosiana Pilsbry 1908

Distribución geográfica: EN. México: Tamaulipas (Tampico), San Luis Potosí (Cd. Valles).

Euglandina sp. nov.

Distribución geográfica: EN. México: San Luis Potosí (Xilitla).

Euglandina texasiana (Pfeiffer 1857)

Distribución geográfica: NTp y NA. Sur de Texas (E. U. A.); México: Nuevo León (El Diente y Santiago), Tamaulipas (Tampico), San Luis Potosí (Cd. Valles y El Abra), Veracruz. 1936)

Euglandina texasiana angustior (Pilsbry y Vannata

Distribución geográfica: EN. México: Tamaulipas

(González), San Luis Potosí (Cd. Valles).

Salasiella hinkleyi Pilsbry 1919

Distribución geográfica: EN. México: San Luis Potosí (El Abra, Tamasopo). 
Salasiella sp. nov.

Distribución geográfica: EN. México: San Luis Potosí (Cascadas Micos).

Spiraxis sp. nov.

Distribución geográfica: EN. México: San Luis Potosí (región oriental).

Streptostyla bartschii Dall 1908

Distribución geográfica: EN. México: Tamaulipas (sin localidad específica), San Luis Potosí (Cascadas Micos).

Streptostyla gracilis Pilsbry 1907

Distribución geográfica: EN. México: Tamaulipas

(Tampico), San Luis Potosí (Cd. Valles).

Streptostyla jilitlana Dall 1908

Distribución geográfica: EN. México: San Luis Potosí (Xilitla).

Streptostyla minuta Pilsbry 1909

Distribución geográfica: EN. México: San Luis Potosí (Canoas).

Streptostyla palmeri Dall 1905

Distribución geográfica: EN. México: San Luis

Potosí (Montañas Alvarez).

Streptostyla potosiana Dall 1905

Distribución geográfica: EN. México: San Luis Potosí (Montañas Alvarez)

Stretostyla supracostata Pilsbry 1909

Distribución geográfica: EN. México: San Luis Potosí (Canoas, El Abra y San Dieguito).

Sagdidae

Microconus sp. nov

Distribución geográfica: EN. México: San Luis Potosí (Xilitla).

Thysanophora fuscula (Adams 1849)

Distribución geográfica: NT. México: Tamaulipas

(Cd. Victoria, Tampico); Jamaica.

Thysanophora horni (Gabb 1866)

Distribución geográfica: NAp y NT. Texas, Nuevo México y Arizona (E. U. A.); México: Sonora, Chihuahua, Wuevo León (Iturbide, Topo Chico), Tamaulipas (Cd. Victoria), Baja California Sur, Sinaloa, San Luis Potosí, Jalisco.

\section{Urocoptidae}

Coelocentrum hinkleyi Pilsbry 1909

Distribución geográfica: EN. México: San Luis Potosí (Micos, San Dieguito).

Coelocentrum priosculpta Thompson y Correa 1994

Distribución geográfica: EN. México: Tamaulipas (Santa María Guadalupe, Ocampo), San Luis Potosí (Naranjos, Cd. del Maíz).

Coelocentrum tanydeira Thompson 1968

Distribución geográfica: EN. México: San Luis Potosí (Xilitla).

Eucalodium ischnostele (Pilsbry 1909)

Distribución geográfica: EN. México: San Luis Potosí (Espinazo del Diablo, Micos, Tamasopo).
Holospira hinkleyi Pilsbry 1907

Distribución geográfica: EN. México: San Luis Potosí (Agua Buena, El Abra, Espinazo del Diablo, Tamasopo)

Microceramus mexicanus (Martens 1897)

Distribución geográfica: NTp y NA. México: Nuevo León (El Diente, Río Mauricio, Santiago), Tamaulipas (Cd. Victoria), San Luis Potosí (Agua Buena, Canoas, El Abra), Veracruz (Arroyo Grande, Misantla, Orizaba), Jalisco (Sayula).

Bulimulidae

Drymaeus emeus (Say 1829)

Distribución geográfica: NTp y NA. México: Nuevo León (Iturbide, Santiago), Tamaulipas (Cd. Victoria), Veracruz (Atoyac, Coatepec, Córdova, Cuatitlán, Misantla, Nautla, Papantla), camino de Veracruz a Cd. de México, Puebla (Necaxa), Tabasco (Teapa). Drymaeus multilineatus (Say 1825)

Distribución geográfica: NT. Florida (E.U.A.); México: Campeche (Campeche), Yucatán (Progreso), sureste de México; Centroamérica; Cuba; noreste de Sudamérica.

Drymaeus sulphureus (Pfeiffer 1857)

Distribución geográfica: NT. México: Veracruz (Atoyac, Jalapa, Orizaba, Texolo), Cd. de México, Tabasco (Teapa), Campeche (Campeche y Cd. del Carmen), Yucatán (Libre Unión); Guatemala; Nicaragua.

Orthalicus princeps (Sowerby 1833)

Distribución geográfica: NT. México: Tamaulipas (Tampico), San Luis Potosí (Cd. Valles), Veracruz (Atoyac, Arroyo Hueyapam, Callejones de Malibrán, Coatepec, Jalapa, Mirador, Misantla, Papantla, Playa Vicente, Veracruz), Sinaloa (Mazatlán), Islas Marías, Colima, Guerrero (Ixtapa), Oaxaca (Tuletepec), Chiapas (Escuintla), Campeche (Champotón, Campeche, Hopelchén, Dzibalchén, Cayal), Yucatán (Actua Has, Aguada, Chichen- itzá, Cenote Ixil, Shkolak, Silam, Sitilpech, Tabi, Ticul, Tunkas), Quintana Roo (Is. Cozumel); áreas tropicales de México y Centroámerica. Rabdotus alternatus (Say 1830)

Distribución geográfica: PA. Sureste de Texas, Louisiana (E. U. A.); México: Chihuahua, Coahuila, Nuevo León (Iturbide, Santiago), Tamaulipas (Cd. Victoria), Durango, San Luis Potosí (Charco Blanco, Cd. Valles), sur y norte de Veracruz, Jalisco, Puebla (Pico de Orizaba), Oaxaca (Tehuantepec).

\section{Polygyridae}

\section{Polygyra cereolus carpenteriana (Bland 1860)}

Distribución geográfica: NT. Florida (E. U. A.); México: Yucatán (Chichen-Itzá, Cerro Isla Ciénega, Mérida, Progreso), Quintana Roo (Is. Mujeres, Puerto Morelos), este de México.

Polygyra dorfeuilliana Lea 1838

Distribución geográfica: NAp y NT. Centro y sur de E. U. A.; México: San Luis Potosí (Cd. Valles). 
Polygyra implicata (Martens 1865)

Distribución geográficá: NTp y NA. Sureste de Texas (E. U. A.); México: Nuevo León (Santiago), Tamaulipas (Magitcatzin, Río Tamesí, Villagrán), San Luis Potosí (Agua Buena, Canoas, Micos, Río Panuco, San Dieguito, Tamazunchale, Valles), Veracruz, Oaxaca.

Polygyra oppilata (Morelet 1849)

Distribución geográfica: NTp y NA. Sureste de Texas (E. U. A.); México: Tamaulipas (González, Tampìco), San Luis Potosí (Río Pánuco, Valles), Veracruz, Oaxaca (Tehuantepec), Yucatán.

Polygyra texasiana (Moricand 1833)

Distribución geográfica: PA. Sur de E. U. A.; México: Nuevo León (Cerralvo), Tamaulipas, San Luis Potosí (Cd. Valles); Veracruz.

Praticolella berlandieriana (Moricand 1833)

Distribución geográfica: NAp y NT. Texas y Ařkansas (E. U. A.); México: Nuevo León (Iturbide, Monterrey, Pablillo, Santa Barbara, Santiago, Topo Chico), Tamaulipas (Cd. Victoria), Durango (Ventanas), San Luis Potosí (Cd. Valles), Veracruz.

Praticolella martensiana (Pilsbry 1907)

Distribución geográfica: EN. México: Tamaulipas (Tampico), San Luis Potosí (Agua Buena, Canoas, El Abra, Cd. Valles).

Xanthonycidae

Trichodiscina cordovana (Pfeiffer 1858)

Distribución geográfica: NTp y NA. México: Nuevo Zeón (Diente, Santiago), Tamaulipas (Cd. Victoria, ampico), Veracruz (Almolonga, Barranca de Teocelo, Cerro de Espaidilla, Coatepec, Consolapa, Córdova, Chirimoyo, Dos Arroyos, Jalapa, Mirador, Misantla, Nacimiento de Quelite, Nautla, Rancho de Guerrero, Soledad, Tuxpan), Guerrero (Omilteme), Oaxaca.

\section{DISCUSIÓN}

Qi principal componente zoogeográfico fue el endemismo del $32.18 \%$ (28 especies y tres subespecies) de la fauna malacológica terestre (Cuadro 1). Le siguió en importancia el componente neotropical del $27.59 \%$ (24 especies y cinco subespecies) de la fauna. El $17.24 \%$ (15 especies y dos subespecies) fue aeotropical principalmente y neártica y el $12.64 \%$ (11 especies) fue de distribución panamericana. El $9.20 \%$ (ocho especies y una subespecie) presentó distribución neártica principalmente y neotropical. Sólo una especie (1.15\%) fue exclusivamente neártica, con base en la distribución conocida. El endemismo en el área de estudio resultó mayor al hallado por
Pilsbry (1903) y Correa (1996-1997) en otras zonas del noreste de México.

La familia con más endemismos fue Spiraxidae con 16 especies y una subespecie (94.12\% en relación con el número total de especies de la familia en el área de estudio). Le siguió Urocoptidae con cinco especies (83.33\%), (Cuadro 2).

En el oriente de San Luis Potosí se conocen 28 especies endémicas, por lo que es una de las zonas en el noreste de México con mayor importancia biogeográfica en cuanto a malacofauna terrestre. En el centro y sur de Tamaulipas se conocen hasta ahora 19 especies endémicas (Correa 1992, Thompson y Correa 1994), muchas de ellas compartidas con el este de San Luis Potosí. La mayor importancia biogeográfica del área de estudio quizás se deba a que es una de las dos zonas en la región noreste de México que han sido revisadas con mayor profundidad (Correa et al.1998).

Al aplicar criterios estrictos para determinar la distribución geográfica, algunas especies pueden catalogarse como principalmente neotropicales, y en menor grado neárticas (Microceramus mexicanus). Sin embargo, debe considerarse al centro de Nuevo León (al norte del Trópico de Cáncer), zona donde están presentes también otras especies (Drymaeus emeus, Trichodiscina cordovana, Helicina sowerbyana y Habroconus elegantula), como una zona de transición neártica - neotropical (Correa 1996-1997).

Ochenta especies $(91.95 \%$ del total de especies) de caracoles terrestres están presentes en la zona montañosa de la región central del área de estudio. Por lo anterior, es muy probable que esta zona de la Sierra Madre Oriental funcione como un centro de dispersión de especies en la región.

La mayor cantidad de especies de gastrópodos terrestres en el noreste y el este de México se conocen o se han descrito principalmente en localidades de la Sierra Oriental (El Diente, oeste de Cd. Victoria, Necaxa, Xalapa, Tehuacán), otras provincias montañosas como el Eje Neovolcánico Transversal y la Sierra Madre del Sur, o en los límites de algunas de éstas con las Llanuras Costeras del 


\section{CUADRO 1}

Distribución geográfica de las especies de gastrópodos terrestres en la Huasteca Potosina $(N=87)$

Distribución geográfica

Porcentaje

Especies endémicas

Especies con distribución exclusivamente neotropical

Especies con distribución neotropical principalmente y alguna presencia en el neártico

Especies con distribución panamericana

Especies con distribución neártica principalmente y alguna presencia en el neotrópico

Especies con distribución exclusivamante neártica

\section{CUADRO 2}

Familias de gastrópodos terrestres, número de especies endémicas y su porcentaje respecto a la cantidad total de especies por familia en la región oriental de San Luis Potosí, México.

Familia

Especies endémicas

Porcentaje

Helicinidae

Succineidae

Zonitidae

Spiraxidae

Sagdidae

Urocoptidae

Polygyridae

2
1
2
16
1
5
1

22.22

33.33

40.00

94.12

33.33

83.33

14.28

Golfo Norte y del Golfo sur (Misantla, Córdova, Orizaba) (Baker 1930, Martens 1890, Pilsbry 1899,1903$)$. Esto significa que a una escala geográfica mayor, la Sierra Madre Oriental es el centro de dispersión de las especies en el este y noreste de México.

Las especies con la distribución conocida más amplia hacia el sur en los trópicos hasta Sudamérica fueron Pupisoma dioscoricola insigne (Centro y Sudamérica), Lamellaxis micra (Centroamérica, Venezuela, Brasil y Bolivia), Beckianum beckianum (Centroamérica, Brasil y Ecuador), Guppya gundlachi (Cen- troamércia, Venezuela y Brasil) y Gastrocopta pellucida (Centroamérica y Ecuador). Rumina decollata, Zonitoides arboreus, Hawaiia minuscula, Lamellaxis gracilis y Subulina octona fueron las especies presentes en el oriente de San Luis Potosí que tuvieron la más amplia distribución mundial conocida.

\section{AGRADECIMIENTOS}

A Fred G. Thompson del Florida Museum of Natural History, University of Florida por la literatura proporcionada y los comentarios al estudio. A Artie L. Metcalf de la University of Texas at El Paso por la revisión detallada del trabajo y los comentarios sobre algunas especies. A Antonio García Cubas y Martha Reguero por la revisión crítica del manuscrito y sus sugerencias. Al Consejo Nacional de Ciencia y Tecnología por el apoyo económico. A Rubén Rodríguez por su participación a lo largo de esta investigación.

\section{RESUMEN}

Se estudiaron (Junio, 1995 a Agosto, 1996) los caracoles terrestres de la región oriental de San Luis Potosí, México. Esta región posee una gran variación topográfica y climática. Los muestreos (161) se realizaron en 48 localidades. Los principales componentes zoogeográficos de los gastrópodos terrestres fueron el endémico (32.18\%: 28 especies y tres subespecies) y el neotropical (27.59 \%: 24 especies y cinco subespecies). Spiraxidae fue la familia con más endemismos (16). La Sierra Madre Oriental posee el mayor número de especies en la región.

\section{REFERENCIAS}

Anónimo. 1980. Carta de uso de suelo y vegetación. Instituto Nacional de Estadística, Geográfica e Informática (INEGI). Esc. $1: 1000,000$. México, D.F.

Anónimo. 1981a. Carta fisiográfica. Instituto Nacional de Estadística, Geográfica e Informática (INEGI). Esc. $1: 1000,000$. México, D. F:

Anónimo. 1981b. Carta de climas. Instituto Nacional de Estadística, Geográfica e Informática (INEGI). Esc. $1: 1000,000$. México, D.F.

Baker, H. B. 1922. The mollusca collected by University of Michigan-Walker Expedition in Southern 
Veracruz, México. Part I Occas. Pap. Mus. Zool. Univ. Mich. 106: 1-95.

Baker, H. B. 1923. The mollusca collected by The University of Michigan - Walker Expedition in Southern Veracriz, México. Part. IV. Occas. Pap. Mus. Zool. Univ. Mich. $135: 1-18$.

Baker, H. B. 1928. Mexican mollusks collected for Dr. Bryant Walker in 1926. Part. I. Occas. Pap. Mus. Zool. Univ. Mich. $193: 1-65$.

Baker, H. B. 1930. Mexican mollusks collected for Dr. Bryant Walker in 1926. Part. II. Occas. Pap. Mus Zool. Univ. Mich. $220: 1-45$.

Bequaert, J. C. \& W. B. Miller. 1973. The mollusks of the arid southwest with an Arizona check list. University of Arizona, Tucson, Arizona. p. 12 - 13.

Cameron, R.A.D. \& M. Redfern. 1976. A synopsis of the british land snails. Academic, Londres: 9

Coney, C., W. A. Tarpley \& R. Bohannan. 1981. A metod of collecting minute land snails. Nautilus 95: $43-$ 44.

Correa, S. A. 1992. Diversidad, distribución y especies aprovechables como alimento de la malacofauna terrestre del centro y sur de Tamaulipas, México. Informe de proyecto. Consejo Tamaulipeco de Ciencia y Tecnología. Cd. Victoria, Tamps. 81 p.

Correa, S. A. 1993. Caracoles terrestres (Mollusca: Gastropoda) de Santiago, Nuevo León, México. Rev. Biol. Trop. $41: 683-687$.

Correa, S. A. 1996-1997. Caracoles terrestres (Mollusca: Gastropoda) de Iturbide, Nuevo León, México. Rev. Biol. Trop. 44/45 : 137 - 142.

Correa S. A., A. G. Cubas \& M. M. Reguero. 1998. Gastrópodos terrestres de la región oriental de San Luis Potosí, México. Acta Zool. Mex. (n. s.) 73: 1 - 17.

Cheatum, E. P. \& R. W. Fullington. 1971. The aquatic and land mollusca of Texas. I. The recent and pleistocene members of the gastropod family Polygyridae in Texas. Dall. Mus. Nat. Hist. Bull. 1. 61 p.

Cheatum, E. P. \& R. W. Fullington. 1973. The aquatic and land mollusca of Texas. II. The recent and pleistocene me bers of the Pupillidae and Urocoptidae (Gastropoda) in Texas. Dall. Mus. Nat. Hist. Bull. I. 67 p.

Fischer, P. \& H. Crosse. 1870 - 1902. Etude sur les mollusques et fluviatiles du Mexique et du Guatemala.
Miss. Scient. au Mexique et dan's Amerique Centrale. Recherches Zoologiques. Pt. 7.1 - 2: 702 -731 .

Hubricht, L. 1985. The distribution of the native land mollusks of the eastern United States. Field Zool. 24 : 1-191.

Lavelle, P., M. E. Maury \& V. Serrano. 1981. Estudio cuantitativo de la fauna del suelo en la región de Laguna Verde, Ver cruz. Epoca de lluvias, p. 73 105. In. Reyes-Castillo, P. (ed.) Estudios ecológicos en el trópico mexicano. Inst. Ecol., A. C. 6.

Martens, E. V. 1890. Biologia Centrali -Americana. Land and fresh water moll sca. Taylor \& Francis. Londres. 706 p.

Peake, J. 1978. Distribution and ecology of the Stylommatophora : 430 - 495. In Fretter V. y J. Peake (ed.). Pulmonates. 2A. Systematics, evolution and ecology. Academic, Londres.

Pérez, A. M. \& A. López. 1993. Nuevos registros para la fauna malacológica terrestre y fluviatil de Nicaragua. Rev. Biol. Trop. 41: 913-915.

Pérez, A. M., J. C. Vilaseca \& N. Zione. 1996. Sinecología básica de moluscos terrestres en cuatro formaciones vegetales de Cuba. Rev. Biol. Trop. 44: 133-146.

Pilsbry, H. A. 1899. Descriptions of new species of Mexican land and freshwater mollusks. Proc. Acad. Nat. Sci. Phil. 32: 391-402.

Pilsbry H. A. 1903. Mexican land and freshwater mollusks. Proc. Acad. Nat. Sci. Phil. 34: 761-789.

Pilsbry H. A. 1919. Mollusca from Central America and México. Proc. Acad. Nat. Sci. Phil. 71: 212-223.

Pilsbry H. A. 1939. Land mollusca of North America (North of México). Monog. Acad. Nat. Sci. Phil. 1: 1-574.

Pilsbry H. A. 1940. Land mollusca of North America (North of México). Monog. Acad. Nat. Sci. Phil. 2: 575-994.

Pilsbry H. A. 1946. Land mollusca of North America (North of México). Monog. Acad. Nat. Sci. Phil. 1: 1-520.

Pilsbry H. A. 1948. Land mollusca of North America (North of México). Monog. Acad. Nat. Sci. Phil. 2: 521-1113.

Pilsbry H. A. 1953. Inland mollusca of northern México. II. Urocoptidae, Pupillidae, Strobilopsidae, 
Valloniidae and Cionellidae. Proc. Acad. Nat. Sci. Phil. 105: 133-167.

Pilsbry H. A. 1956. Inland mollusca of northern México. III. Polygyridae and Potadominae. Proc. Acad. Nat. Sci. Phil. 108: 19-40.

Rzedowski, J. 1978. Vegetación de México. Limusa, México, D. F. 432 p.

Solem, A. 1956. The helicoid cyclophorid mollusks of México. Proc. Acad. Nat. Sci. Phil. 108: 41-59.

Solem, A. 1957. Notes on some mexican land snails. Notulae Naturae 298: 1-13.

Taylor, D. W. \& N. F. Sohl. 1962. An outline of gastropod classification. Malacologia 1: 7-32.
Thompson, F. G. 1968. Some mexican land snails of the family Urocoptidae. Bull. Fla. State. Mus. 12: 125183.

Thompson F. G. 1980. Proserpinoid land snails and their relationships within the Archaeogastropoda. Malacologia 20: 1-33.

Thompson F. G. \& A. Correa S. 1994. Land snails of the genus Coelocentrum from northeastern México. Bull. Fla. Mus. Nat. Hist., Biol. Sci. 36: 141-173.

Zilch, A. 1959-1960. Gastropoda. Teil 2, Euthyneura. In. Schindewolf, Handbuch der Palaeozoologie, v. 6, Borntraeger. Berlin. Xii +834 p. 